Check for updates

Cite this: RSC Adv., 2017, 7, 50425

Received 18th September 2017 Accepted 20th October 2017

DOI: 10.1039/c7ra10363h

\section{Construction of poly(dopamine) doped oligopeptide hydrogel $\uparrow$}

\author{
Chen-Ming Zhang, Si-Yong Qin, (D) * Yin-Jia Cheng and Ai-Qing Zhang*
}

Incorporation of poly(dopamine) (PDA) into the native oligopeptide hydrogel has been demonstrated to decrease the critical gel concentration (CGC), improve the rigidity of formed hybrid hydrogel, and especially endow the hydrogel with efficient free radical scavenging ability.

rsc.li/rsc-advances

Inspired by nature, self-assembly strategy has been well developed to build various biomaterials in recent years. For example, self-assembly of DNA, ${ }^{1,2}$ phospholipids, ${ }^{3,4}$ proteins, ${ }^{5,6}$ as well as peptides $^{7}$ has been widely exploited to construct different nanomaterials for drug/gene delivery, cell culture and tissue engineering. Among them, the oligopeptides with low molecular weights have attracted much attention in the field of nanotechnology-assistant cancer treatments due to the mellow synthetic methods and adjustable amphipathy to fabricate shape-tailored nanomaterials via self-assembly. Of note, selfassembled oligopeptide hydrogel has been demonstrated as an interesting soft material for biomedical applications. ${ }^{8,9}$ Nevertheless, restricted by the short sequences, most oligopeptides are lack of multiple bioactivity. On account of the noncovalent interactions, in addition, the hydrogel strength is usually far from satisfactory. ${ }^{10,11}$

To tackle these difficulties, various hybrid oligopeptide hydrogels have been developed to hold different synergistic properties. Incorporation of graphene oxide (GO) into a short peptide hydrogel improved the rigidity of the hybrid hydrogel system compared to the native one. ${ }^{12}$ The hybrid hydrogel constructed by GO sheets and an oligopeptide possessed high drug-sustainability and on-demand drug release in vivo. ${ }^{13}$ Doping FEFEFKFK peptide hydrogel with $\operatorname{poly}(N$ isopropylacrylamide)-FEFEFKFK conjugate allowed for optimizing the mechanical properties of peptide-based hydrogels and endowed them with temperature sensitivity. ${ }^{14}$ Hybrid hydrogel compromising of an amphipathic oligopeptide and a fluorescent $\pi$-conjugated poly(para-phenylene vinylene) exhibited strong fluorescence, even higher than the pure polymer in solution. ${ }^{15}$ As anticipated, different functions have been achieved in those two-in-one hybrid systems. Nevertheless, the

College of Chemistry and Materials Science, South-Central University for Nationalities, Wuhan 430074, P. R. China. E-mail: sy-qin@mail.scuec.edu.cn; aizhang@scuec.edu.cn

$\dagger$ Electronic supplementary information (ESI) available: Experimental details about the synthesis and characterization of poly(dopamine), oligopeptide, and oligopeptide hybrid hydrogel. See DOI: 10.1039/c7ra10363h controversy on the toxicity of GO in vivo slows its progress toward clinical biomaterials. ${ }^{16-18}$ And preparing functional polymers sometimes needs tedious steps. Thus, functional polymers with good biocompatibility and simple fabrication process are highly pursued to construct novel hybrid oligopeptide hydrogels.

Poly(dopamine) (PDA) owing to many functional groups such as catechol, amine, and imine exhibits robust adhesion to diverse substrates, which provides great potential as the coating materials. ${ }^{19,20}$ It has been well documented that PDA could adsorb various dye molecules due to the hydrogen bonding and $\pi-\pi$ stacking interaction. ${ }^{21,22}$ Recently, PDA has caused serious concerns in biomedical applications due to the outstanding biocompatibility and unique physicochemical properties..$^{23,24}$ PDA has been demonstrated to be an efficient antioxidant in the biological system, which is associated with the striking properties of sequestering redox active metal ions ${ }^{25,26}$ and scavenging reactive free radicals..$^{27,28}$ Specially, PDA-bearing hybrid hydrogels with specific functionalities have been developed, in which PDA acts as cross-linking mediator and the yielded hybrid hydrogel can be used in biomedical field as drug delivery carriers and tissue adhesives. ${ }^{29}$

In the present study, a novel hybrid peptide hydrogel was fabricated by incorporating PDA into native oligopeptide hydrogel (Scheme 1). The intermolecular interaction between the Fmoc appended oligopeptide and PDA was investigated. Incorporation of PDA into native oligopeptide hydrogel can decrease critical gel concentration (CGC) and improve the rigidity of the formed hydrogel. Specially, the novel hybrid peptide exhibits a combinational feature of peptide-based species and PDA materials, which shows great potential in the fields of medical wound dressing and drug control release.

PDA was synthesized by the autopolymerization of dopamine in an aerobic atmosphere under alkaline situation. The oxidation and polymerization process was monitored by fluorescent spectroscopy, accompanying with the color change of the solution. Along with the increase in reaction time, the colorless solution became brown and even black, demonstrating the successful oxidation of dopamine in basic condition (see 


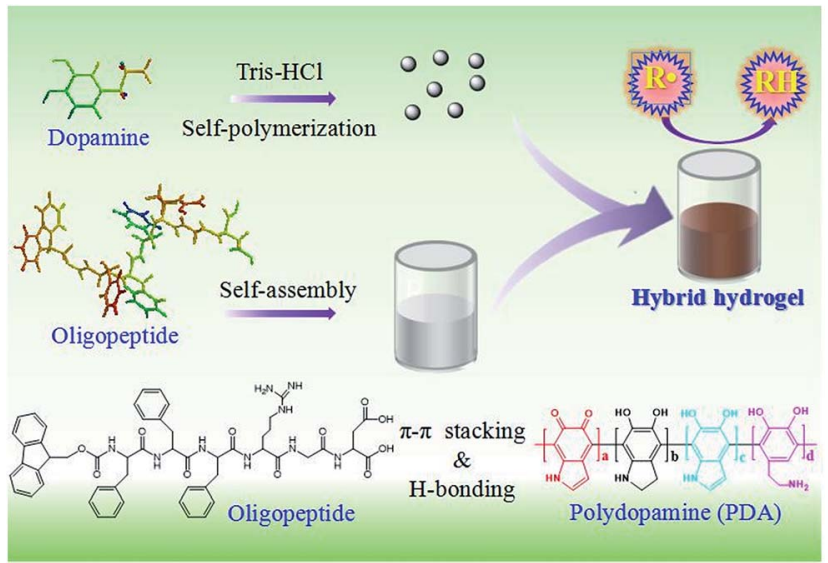

Scheme 1 Formation of mechanism of PDA doping hybrid oligopeptide hydrogel. $\mathrm{R}^{*}$ and $\mathrm{RH}$ representing the radical and it corresponding reduced species, respectively.

Fig. S1A in ESI $\dagger$ ). To monitor the oxidation process, the fluorescent spectra of PDA solutions were recorded at different time intervals up to $360 \mathrm{~min}$. Two fluorescent emission peaks at $470 \mathrm{~nm}$ and $495 \mathrm{~nm}$ appeared immediately (see Fig. S1B in ESI $\dagger$ ), which were corresponding to the intermediate and oxidized products, respectively. The appearance of fluorescent peaks suggested the rapid oxidation reaction of dopamine molecules. Interestingly, the intensity ratio between $495 \mathrm{~nm}$ and $470 \mathrm{~nm}$ (marked as $I_{495} / I_{470}$ ) increased in the first $15 \mathrm{~min}$. The reason was attributed to the concurrent formation and consumption of intermediate products in the polymerization process. After $30 \mathrm{~min}$, only an intense peak around $495 \mathrm{~nm}$ was observed. Specially, the emission intensity maxima at $495 \mathrm{~nm}$ started to decrease when the oxidation time exceeded $30 \mathrm{~min}$. This phenomenon might be originated from the self-quench of PDA. The final wavelength with highest fluorescent intensity was determined at $\sim 495 \mathrm{~nm}$, which was in accordance with the result reported by Wei's group, ${ }^{30}$ but different from the wavelength at around $470 \mathrm{~nm}$ obtained by Yildirim and co-workers. ${ }^{31}$ The difference in the maximum emission wavelength resulted from the varying degrees of oxidation. To investigate the morphology of PDA, scanning electron microscopy (SEM) was exploited to image the nano-shape, which disclosed that PDA existed in a regular spherical structure with an average size of $250 \mathrm{~nm}$ (see Fig. S1C in ESI $\dagger$ ). Dynamic light scattering (DLS) profile revealed that the average size of PDA is $270 \mathrm{~nm}$ (see Fig. S1D in ESI $\dagger$ ), almost consistent with the SEM result.

The oligopeptide gelator named as Fmoc-FFFRGD-OH was synthesized on 2-chlorotrityl chloride resin using a standard Fmoc chemistry according to the literature procedure,,$^{32,33}$ and the molecular structure, molecular weight and purity were characterized, accordingly (see Fig. S2-S5 in ESI $\dagger$ ). The gelation ability of the native oligopeptide was inspected through the vial inversion test. As shown in Fig. 1, the oligopeptide molecules aggregated into macroscopical hydrogel when the peptide concentration reached $7 \mathrm{mg} \mathrm{mL} \mathrm{mL}^{-1}(0.7 \mathrm{wt} \%)$. The formation of the peptide hydrogel resulted from the molecular self-assembly. To investigate the influence of PDA on gelation ability of the

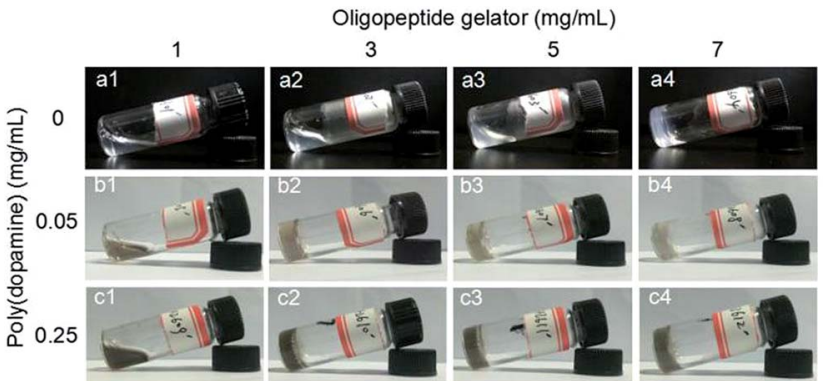

Fig. 1 Photos of native oligopeptide and PDA doped hybrid hydrosol/ hydrogel obtained from different peptide and PDA concentrations.

peptide, different amounts of PDA were added. Notably, the oligopeptide with a concentration of $3 \mathrm{mg} \mathrm{mL} \mathrm{mL}^{-1}(0.3 \mathrm{wt} \%)$ can self-assemble into hydrogel with the help of $0.05 \mathrm{mg} \mathrm{mL}^{-1}$ PDA nanoparticles (Fig. 1), which was apparently lower than $7 \mathrm{mg} \mathrm{mL} \mathrm{m}^{-1}(0.7 \mathrm{wt} \%)$ required for the native peptide hydrogel. Meanwhile, an interesting phenomenon occurred once changing the concentration of PDA nanoparticles. Improving the PDA concentration to $0.25 \mathrm{mg} \mathrm{mL}^{-1}$ enhanced the rigidity of the hybrid hydrogel, comparing with the hydrogel in the presence of $0.05 \mathrm{mg} \mathrm{mL}^{-1}$ PDA nanoparticles. But further elevating the PDA concentration to $1.25 \mathrm{mg} \mathrm{mL}^{-1}$, the oligopeptide could not self-assemble into hydrogel at $3 \mathrm{mg} \mathrm{mL}^{-1}$ and a higher concentration of $5 \mathrm{mg} \mathrm{mL}{ }^{-1}$ was needed (see Fig. S6 in ESI $\dagger$ ). Increasing the PDA concentration to $2.50 \mathrm{mg} \mathrm{mL}^{-1}$, the oligopeptide could not form hybrid hydrogel in the observed concentration range. The reason was as following: PDA nanoparticles could act as cross-linking mediator, which interacted with the oligopeptide gelator and therefore facilitated the selfassembly. But excessive PDA nanoparticles would hinder the effective arrangement of peptide molecules and led to the failure of hydrogel. Meanwhile, the stability of the oligopeptide hydrogel was evaluated using thermogravimetric analysis (TGA). As shown in Fig. S7, $\uparrow$ about $12 \%$ weight loss occurred between 180 and $220{ }^{\circ} \mathrm{C}$, which might be attributed to partial thermal decomposition of dry gel. Another major weight loss of $25 \%$ was observed in the temperature interval from $270{ }^{\circ} \mathrm{C}$ to $390{ }^{\circ} \mathrm{C}$, which was caused by completed thermal degradation of oligopeptide. ${ }^{33}$

Morphologies of native oligopeptide hydrogel and hybrid hydrogel were imaged by SEM. SEM image of the diluted oligopeptide hydrogel is shown in Fig. S8 (ESI $\dagger$ ), indicating the entangled nanofibrillar structure. Furthermore, the morphology of hybrid hydrogel was also observed by SEM, which revealed the co-existence of both PDA nanoparticles and oligopeptide nanofibers. Interestingly, the PDA nanoparticles were embedded in the peptide hydrogel matrix, indicating the potential interaction between PDA nanoparticles and peptide nanofibers.

To study the intermolecular interaction in the native hydrogel and hybrid hydrogel, FT-IR experiment was carried out to probe the intermolecular hydrogen bonding. As shown in Fig. 2A, the amide A band (NH stretching) at around $3230 \mathrm{~cm}^{-1}$ instead of around $3400 \mathrm{~cm}^{-1}$ was observed, in the backbone 
were involved in hydrogen bonding formation in the native hydrogel. ${ }^{12}$ The appearance of the bands at $1632 \mathrm{~cm}^{-1}$ (amide I) and $1550 \mathrm{~cm}^{-1}$ (amide II) indicated the presence of $\beta$-sheet conformation in the hydrogel. ${ }^{34}$ Meanwhile, the FT-IR spectrum of PDA was also recorded and a band centered at around $1630 \mathrm{~cm}^{-1}$ was found, arising from the oxidation of phenolic hydroxyl group of dopamine into keto group. The band at $3400 \mathrm{~cm}^{-1}$ was attributed to the $\mathrm{N}-\mathrm{H}$ stretching vibration of PDA. In addition, PDA-containing hybrid hydrogel showed the similar characteristic bands with that of native oligopeptide hydrogel. This suggested that the $\beta$-sheet conformation was preserved even after the incorporation of PDA into the oligopeptide hydrogel system.

Solvent and concentration dependent fluorescence experiments of oligopeptide were carried out in hydrosol phase, which could offer useful information about the interaction of the
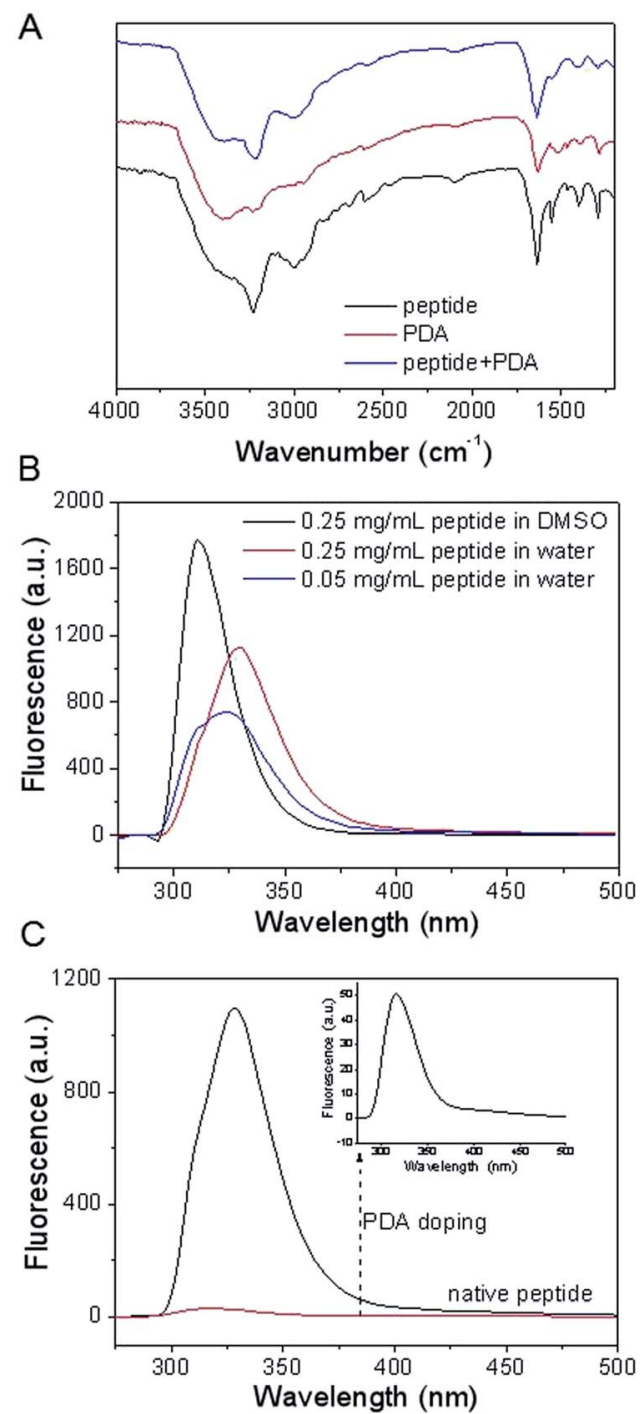

Fig. 2 (A) FT-IR spectra of the lyophilized peptide, PDA and peptide + PDA. (B) Fluorescence spectra of peptides with different concentrations in DMSO and water. (C) Fluorescence spectra of native peptide and PDA containing peptide solutions. aromatic moieties in the gelator molecules. As shown in Fig. 2B, the fluorescent spectrum of $0.25 \mathrm{mg} \mathrm{mL} \mathrm{m}^{-1}$ oligopeptide exhibited an emission peak at $311 \mathrm{~nm}$ in DMSO, where the oligopeptide molecules were expected to exist in a monomeric form. Interestingly, an emission centered at $324 \mathrm{~nm}$ was observed in the fluorescent spectrum of $0.05 \mathrm{mg} \mathrm{mL}^{-1}$ oligopeptide in water, suggesting that the occurrence of selfaggregation of oligopeptide at a very low concentration. The peak at $324 \mathrm{~nm}$ exhibited further red-shift to $340 \mathrm{~nm}$ as the peptide concentration up to $0.25 \mathrm{mg} \mathrm{mL}^{-1}$. The observed redshift of fluorescence emission maximum from $311 \mathrm{~nm}$ to $340 \mathrm{~nm}$ suggested the contribution of $\pi-\pi$ stacking interaction for oligopeptide self-assembly.

To probe the interaction between PDA and oligopeptide hydrogel, the fluorescent spectrum of hybrid hydrosol was also recorded, accordingly. Interestingly, almost all the peptide fluorescence was quenched after the inclusion of PDA into the peptide solution (Fig. 2C). The fluorescent disappearance of original peptide was attributed to the quenching ability of PDA nanomaterials..$^{35,36}$ This suggested the significant interaction between the PDA nanoparticles and the aromatic moieties in the gelator molecules.

To investigate the flow behavior and rigidity of the selfassembled native peptide hydrogel and hybrid hydrogel, small deformation oscillatory measurement was conducted. Fig. 3A revealed that $G^{\prime}$ of $3 \mathrm{mg} \mathrm{mL}{ }^{-1}$ peptide hydrosol exhibited an elastic response with a weak dependence on the testing angular frequency. The $G^{\prime}$ value was greater than $G^{\prime \prime}$ over the entire tested frequency range $\left(0-100 \mathrm{rad} \mathrm{s}^{-1}\right)$, indicating that the peptide sol had a potential gel property and elastic behavior. The similar result of $G^{\prime}>G^{\prime \prime}$ was also observed in $3 \mathrm{mg} \mathrm{mL}^{-1}$ hybrid hydrogel. Interestingly, both the $G^{\prime}$ and $G^{\prime \prime}$ values of hybrid hydrogel were higher than that of pure peptide hydrosol, suggesting that the incorporation of PDA into the native hydrogel would form a more rigid and 'solid-like' material. At a higher weight percent of the PDA $\left(0.50 \mathrm{mg} \mathrm{mL}^{-1}\right), G^{\prime}$ and $G^{\prime \prime}$ values of the hybrid hydrogel increased (Fig. 3B). This result was consistent with the vial inversion test that elevating the PDA content within a certain range was in favour of the formation of hybrid hydrogel. Furthermore, the variation of $G^{\prime}$ and $G^{\prime \prime}$ of native oligopeptide hydrogel $\left(7 \mathrm{mg} \mathrm{mL}^{-1}\right)$ and hybrid hydrogel ( $7 \mathrm{mg} \mathrm{mL}^{-1}$ of peptide and $0.25 \mathrm{mg} \mathrm{mL}^{-1}$ of PDA) was also recorded as a function of applied frequency sweep, which further indicated that the interconnection of PDA and peptide gelator could improve the strength of hydrogel (Fig. 3C).

It has been well documented that phenolic compounds can effectively quench active radicals via an electron transfer process. ${ }^{37,38}$ It is natural to assume that PDA nanoparticles with phenolic groups can scavenge free radicals. 2,2-Diphenyl-1picrylhydrazyl (DPPH) radical scavenging activity of PDA nanoparticles was conducted. As shown in Fig. 4A, the original purple of pure DPPH solution faded once adding the PDA nanoparticles, indicating the rapid capture of free radical from DPPH by PDA nanoparticles. Meanwhile, free radical scavenging activity of PDA nanoparticles exhibited a dose-dependent manner. The quantitative analysis of free radical scavenging activity was conducted by monitoring the decrease in absorbance 


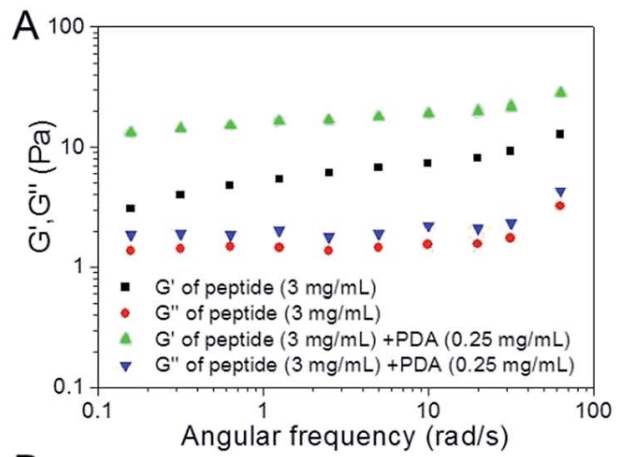

B

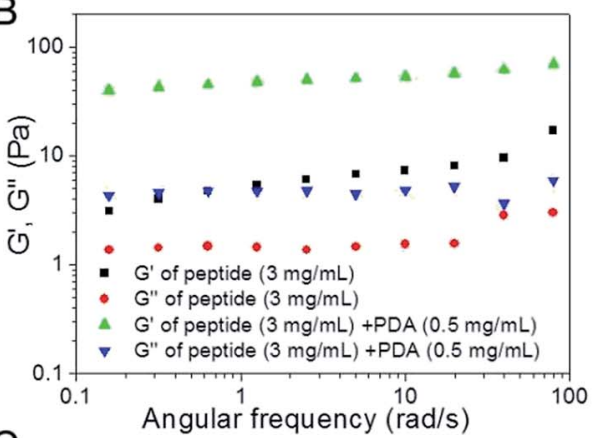

C

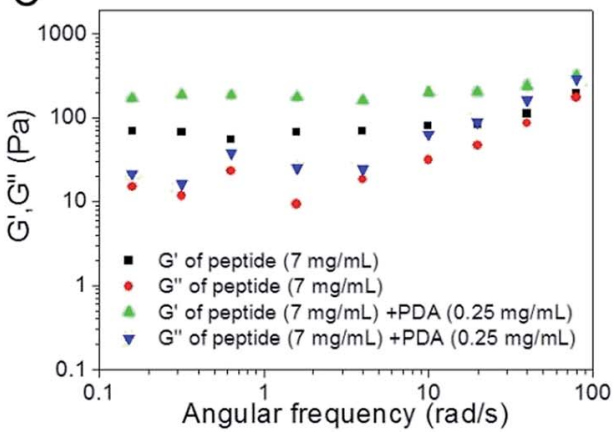

Fig. 3 Frequency dependence of the dynamic storage moduli $\left(G^{\prime}\right)$ and the loss moduli $\left(G^{\prime \prime}\right)$ of native hydrogels and the PDA bearing hybrid hydrogels with different concentrations.

at $516 \mathrm{~nm}$. At a very low PDA concentration of $7.5 \mu \mathrm{g} \mathrm{mL}{ }^{-1}$, $59.2 \%$ of free radical could be captured within $5 \mathrm{~min}$. Notably, $98.2 \%$ of free radicals were scavenged by PDA nanoparticles with a concentration of $15 \mu \mathrm{g} \mathrm{mL} \mathrm{m}^{-1}$ within the same time.

An elevated level of free radical has been implicated in the development and progression of inflammatory response, which is detrimental for the recovery of wound healing. Inflammation is presumably associated, at least in part, with the generated free radical species. ${ }^{39,40}$ To verify the free radical scavenging activity of the hybrid hydrogel, DPPH assay was also carried out, accordingly. Similar with pure PDA nanoparticles, the hybrid oligopeptide hydrogel could also rapidly scavenge the free radical. Using $0.1 \mathrm{mg} \mathrm{mL}^{-1}$ of DPPH as the free radical model, for instance, $86.2 \%$ of free radicals were captured by the hybrid hydrogel in the first 5 min (Fig. 4B). Incubation with hybrid hydrogel for $30 \mathrm{~min}$, nearly $90 \%$ of free radicals were scavenged even at a high concentration of $0.25 \mathrm{mg} \mathrm{mL}^{-1} \mathrm{DPPH}$ (see Fig. S9 in ESI $\dagger$ ). These results showed the great potential of the PDAbearing hybrid hydrogel for anti-inflammation.
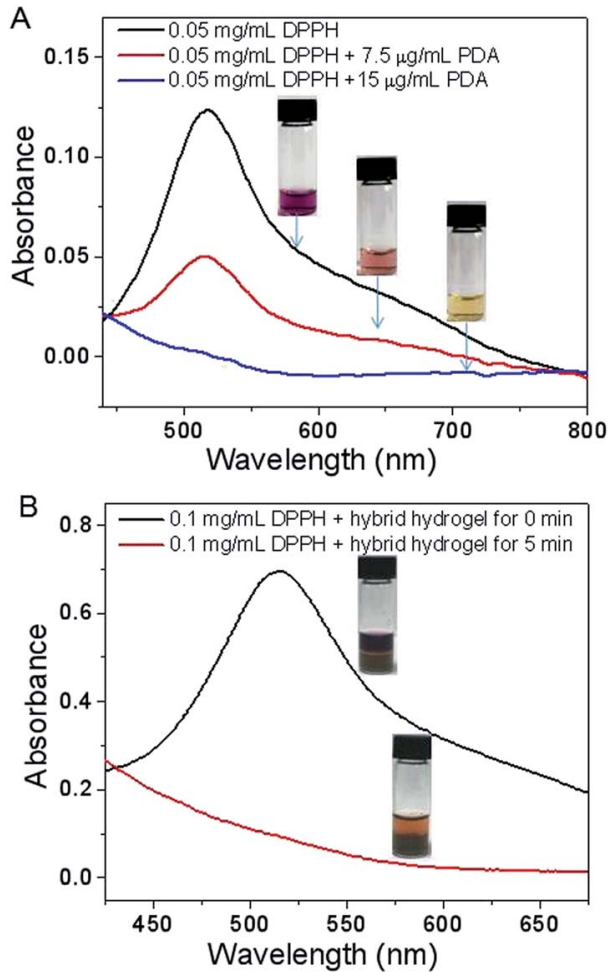

Fig. 4 DPPH radical scavenging activity of synthetic PDA nanoparticles (A) and PDA-bearing hybrid hydrogel (B).

\section{Conclusions}

In summary, a novel hybrid oligopeptide hydrogel with excellent free radical scavenging activity was successfully fabricated by introducing PDA nanoparticles into the native peptide hydrogel. In the presence of PDA as cross-linking mediator, the hybrid hydrogel holds a low CGC value in comparison to native oligopeptide hydrogel. Due to the cooperation of $\pi-\pi$ stacking and hydrogen bonding interactions between aromatic oligopeptide and PDA, the rigidity of the formed hybrid hydrogel has also been improved. Specially, this PDA bearing hybrid hydrogel can efficiently mediate free radical scavenging, which shows great promise for biomedical materials, especially for wound dressing due to the robust adhesion, anti-inflammation, biocompatibility and bioactivity.

\section{Conflicts of interest}

There are no conflicts to declare.

\section{Acknowledgements}

We acknowledge the financial support from the National Natural Science Foundation of China (51503227) and the Natural Science Foundation of Hubei province of China (2017CFB528). 


\section{Notes and references}

1 J. Li, C. Zheng, S. Cansiz, C. Wu, J. Xu, C. Cui, Y. Liu, W. Hou, Y. Wang, L. Zhang, I. Teng, H. H. Yang and W. Tan, J. Am. Chem. Soc., 2015, 137, 1412.

2 Y. Shao, H. Jia, T. Cao and D. Liu, Acc. Chem. Res., 2017, 50, 659.

3 Y. Li, J. Lin, X. Yang, Y. Li, S. Wu, Y. Huang, S. Ye, L. Xie, L. Dai and Z. Hou, ACS Appl. Mater. Interfaces, 2015, 7, 17573.

4 Z. Fan, G. Liu, Y. Li, J. Ma, J. Lin, F. Guo, Z. Hou and L. Xie, RSC Adv., 2016, 6, 82949.

5 X. X. Xia, M. Wang, Y. Lin, Q. Xu and D. L. Kaplan, Biomacromolecules, 2014, 15, 908.

6 Q. Chen, X. Wang, C. Wang, L. Feng, Y. Li and Z. Liu, ACS Nano, 2015, 9, 5223.

7 N. Habibi, N. Kamaly, A. Memic and H. Shafiee, Nano Today, 2016, 11, 41.

8 W. Y. Seow and C. A. E. Hauser, Mater. Today, 2014, 17, 381.

9 L. Sun, C. Zheng and T. J. Webster, Int. J. Nanomed., 2017, 12, 73.

10 D. K. Smith, Nat. Chem., 2010, 2, 162.

11 J. Raeburn, A. Z. Cardoso and D. J. Adams, Chem. Soc. Rev., 2013, 42, 5143.

12 B. Adhikari, J. Nanda and A. Banerjee, Chem.-Eur. J., 2011, 17, 11488.

13 J. Wu, A. Chen, M. Qin, R. Huang, G. Zhang, B. Xue, J. Wei, Y. Li, Y. Cao and W. Wang, Nanoscale, 2015, 7, 1655.

14 A. Maslovskis, J. B. Guilbaud, I. Grillo, N. Hodson, A. F. Miller and A. Saiani, Langmuir, 2014, 30, 10471.

15 J. Rubio-Magnieto, M. Tena-Solsona, B. Escuder and M. Surina, RSC Adv., 2017, 7, 9562.

16 K. Yang, Y. Li, X. Tan, R. Peng and Z. Liu, Small, 2013, 9, 1492.

17 A. B. Seabra, A. J. Paula, R. de Lima, O. L. Alves and N. Durán, Chem. Res. Toxicol., 2014, 27, 159.

18 M. Kryuchkova, A. Danilushkina, Y. Lvov and R. Fakhrullin, Environ. Sci.: Nano, 2016, 3, 442.

19 Z. Wang, C. Li, J. Xu, K. Wang, X. Lu, H. Zhang, S. Qu, G. Zhen and F. Ren, Chem. Mater., 2015, 27, 848.

20 D. Hong, H. Lee, B. J. Kim, T. Park, J. Y. Choi, M. Park, J. Lee, H. Cho, S. P. Hong, S. H. Yang, S. H. Jung, S. B. Ko and I. S. Choi, Nanoscale, 2015, 7, 20149.
21 J. Fu, Z. Chen, M. Wang, S. Liu, J. Zhang, J. Zhang, R. Han and Q. Xu, Chem. Eng. J., 2015, 259, 53.

22 J. Fu, Q. Xin, X. Wu, Z. Chen, Y. Yan, S. Liu, M. Wang and Q. Xu, J. Colloid Interface Sci., 2016, 461, 292.

23 Y. Liu, K. Ai and L. Lu, Chem. Rev., 2014, 114, 5057.

24 M. Liu, G. Zeng, K. Wang, Q. Wan, L. Tao, X. Zhang and Y. Wei, Nanoscale, 2016, 8, 16819.

25 S. Huang, L. Yang, M. Liu, S. L. Phua, W. A. Yee, W. Liu, R. Zhou and X. Lu, Langmuir, 2013, 29, 1238.

26 Y. C. Choi, J. S. Choi, Y. J. Jung and Y. W. Cho, J. Mater. Chem. $B, 2014,2,201$.

27 K. Y. Ju, Y. Lee, S. Lee, S. B. Park and J. K. Lee, Biomacromolecules, 2011, 12, 625.

28 S. Xiong, Y. Wang, J. Yu, L. Chen, J. Zhu and Z. Hu, J. Mater. Chem. A, 2014, 2, 7578.

29 L. Li, W. Smitthipong and H. Zeng, Polym. Chem., 2015, 6, 353.

30 X. Zhang, S. Wang, L. Xu, L. Feng, Y. Ji, L. Tao, S. Li and Y. Wei, Nanoscale, 2012, 4, 5581.

31 A. Yildirim and M. Bayindir, Anal. Chem., 2014, 86, 5508.

32 S. Y. Qin, X. D. Xu, C. S. Chen, J. X. Chen, Z. Y. Li, R. X. Zhuo and X. Z. Zhang, Macromol. Rapid Commun., 2011, 32, 758.

33 S. Y. Qin, Y. Pei, X. J. Liu, R. X. Zhuo and X. Z. Zhang, J. Mater. Chem. B, 2013, 1, 668.

34 M. Baldassarre, M. Bennett and A. Barth, Analyst, 2016, 141, 963.

35 W. Qiang, W. Li, X. Li, X. Chen and D. Xu, Chem. Sci., 2014, 5, 3018.

36 D. Fan, X. Zhu, Q. Zhai, E. Wang and S. Dong, Anal. Chem., 2016, 88, 9158.

37 L. Panzella, G. Gentile, G. D'Errico, N. F. D. Vecchia, M. E. Errico, A. Napolitano, C. Carfagna and M. d'Ischia, Angew. Chem., Int. Ed., 2013, 52, 12684.

38 Y. Liu, K. Ai, X. Ji, D. Askhatova, R. Du, L. Lu and J. Shi, J. Am. Chem. Soc., 2017, 139, 856.

39 T. S. Vo, D. H. Ngo, L. G. Bach, D. N. Ngo and S. K. Kim, Process Biochem., 2017, 54, 188.

40 N. Fujiwara, A. T. Som, L. D. D. Pham, B. J. Lee, E. T. Mandeville, E. H. Lo and K. Arai, Neurosci. Lett., 2016, 633, 7 . 